\title{
Water pressure method for duodenal endoscopic submucosal dissection
}

Duodenal endoscopic submucosal dissection (ESD) is difficult due to insufficient mucosal elevation, because of the coarse submucosal layer [1]. We report on the "water pressure method" for duodenal ESD.

We perform duodenal ESD with a smallcaliber-tip transparent (ST) hood and DualKnifeJ (Olympus, Tokyo, Japan). Insertion of the ST hood under the mucosal flap is a crucial step, as it provides good countertraction to the submucosal layer and good visualization of the operative field. Water pressure by waterjet function of the endoscope helps insertion of the ST hood under the mucosal flap ( Fig. 1; Video 1). Normal saline with a minimum amount of dimethicone is used for the waterjet, rather than water, because normal saline includes electrolytes. With carbon dioxide insufflation, the visual field at the tip of ST hood is small and far. However, water immersion increases the visual field and acts as a magnifier ( $>$ Fig.2). This improved visualization allows the tissue to be cut more precisely. In conclusion, the "water pressure method" is simple and useful for duodenal ESD.

Endoscopy_UCTN_Code_TTT_1AO_2AG

\section{Competing interests}

None

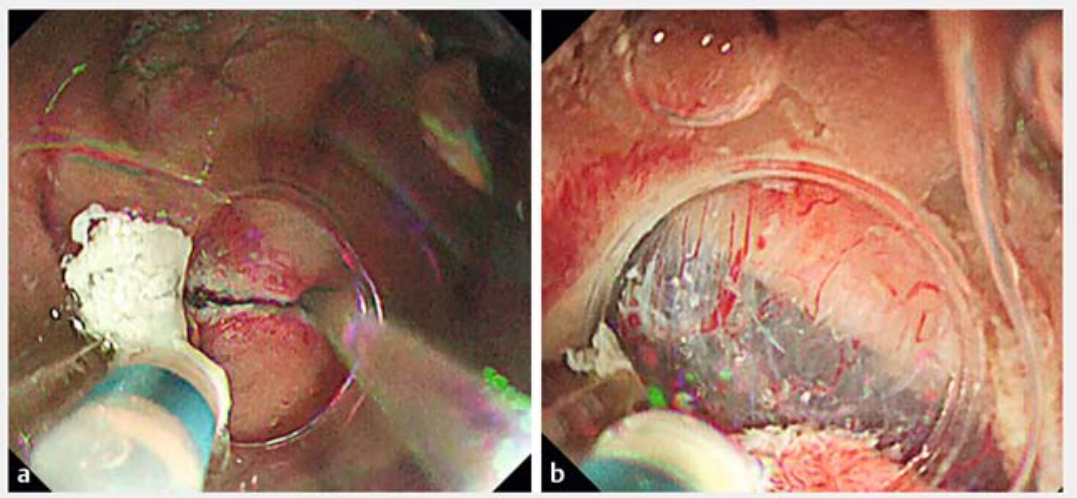

- Fig. 1 The water pressure method for duodenal endoscopic submucosal dissection (ESD). a ESD was performed with a small-caliber-tip transparent (ST) hood and DualKnife] (Olympus, Tokyo, Japan). The waterjet function of the endoscope was used. $\mathbf{b}$ The water pressure helps insertion of the ST hood under the mucosal flap.
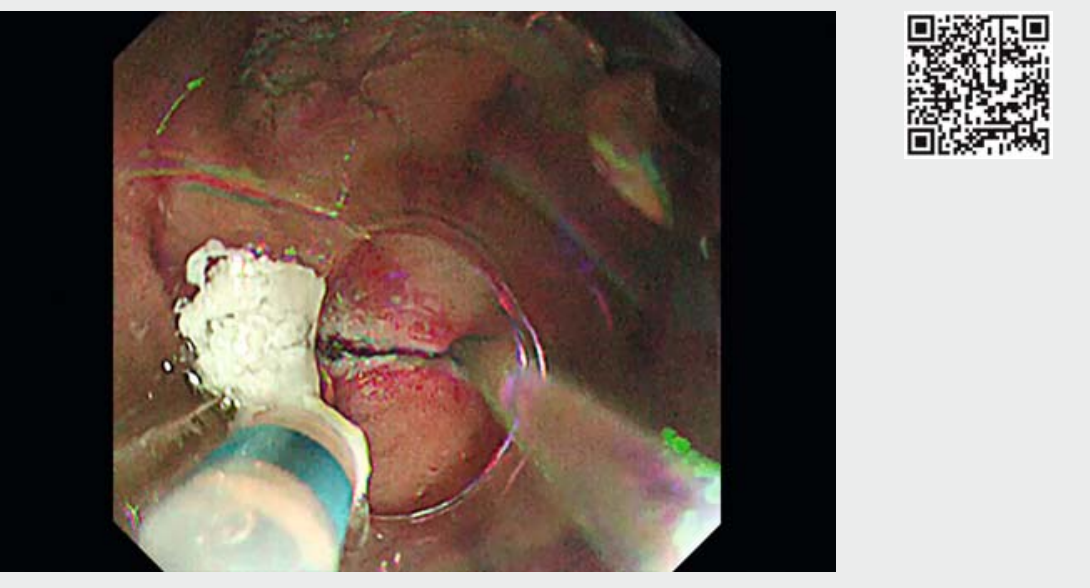

$\checkmark$ Video 1 The water pressure method for duodenal endoscopic submucosal dissection (ESD). ESD was performed with a small-caliber-tip transparent (ST) hood and DualKnife] (Olympus, Tokyo, Japan). The water pressure helps insertion of the hood under the mucosal flap. Water immersion increases the visual field and acts as a magnifier. 


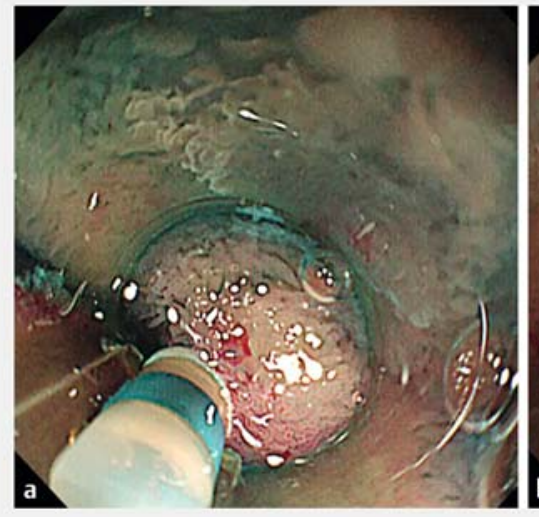

Fig. 2 Water immersion during duodenal endoscopic submucosal dissection. a With carbon dioxide insufflation, the visual field at the tip of the small-caliber-tip transparent hood is small and far. $\mathbf{b}$ Water immersion increases the visual field and acts as a magnifier.

\section{The Authors}

Naohisa Yahagi', Toshihiro Nishizawa 1,2,3, Motoki Sasaki ${ }^{1}$, Yasutoshi Ochiai ${ }^{1}$, Toshio Uraoka ${ }^{1,3}$

1 Division of Research and Development for Minimally Invasive Treatment, Cancer Center, Keio University School of Medicine, Tokyo, Japan

2 Department of Gastroenterology and Hepatology, Keio University School of Medicine, Tokyo, Japan

3 Department of Gastroenterology, National Hospital Organization, Tokyo Medical Center, Tokyo, Japan

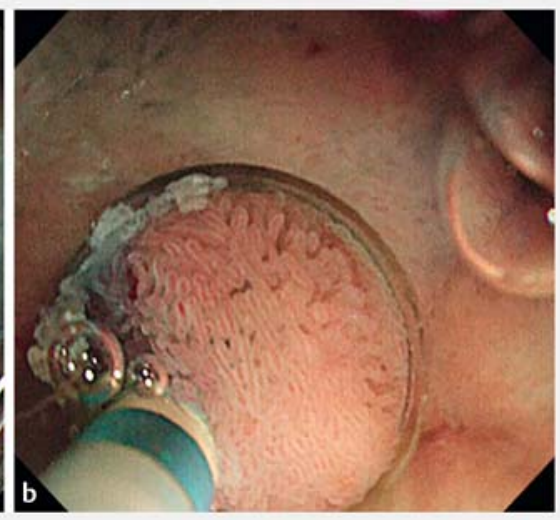

\section{Reference}

[1] Miura Y, Shinozaki S, Hayashi Y et al. Duodenal endoscopic submucosal dissection is feasible using the pocket-creation method. Endoscopy 2017; 49: 8-14

\section{Bibliography}

DOI https://doi.org/10.1055/s-0043-113556

Published online: 31.7.2017

Endoscopy 2017; 49: E227-E228

(c) Georg Thieme Verlag KG

Stuttgart · New York

ISSN 0013-726X

\section{ENDOSCOPY E-VIDEOS}

https://eref.thieme.de/e-videos

\section{Corresponding author}

\section{Naohisa Yahagi, MD, PhD}

Division of Research and Development for Minimally Invasive Treatment, Cancer

Center, Keio University School of Medicine, 35 Shinanomachi, Shinjuku-ku, Tokyo 1608582, Japan

Fax: 81-3-53633967

yahagi-tky@umin.ac.jp 